OPEN ACCESS

Edited by:

Riccardo Schiavina,

University of Bologna, Italy

Reviewed by: Ugo Giovanni Falagario,

University of Foggia, Italy

Riccardo Bartoletti,

University of Pisa, Italy

Andrea Benedetto Galosi,

Marche Polytechnic University, Italy

${ }^{*}$ Correspondence:

Mike Wenzel

Mike.Wenzel@kgu.de

Specialty section:

This article was submitted to

Genitourinary Oncology,

a section of the journal

Frontiers in Oncology

Received: 22 March 2021 Accepted: 18 May 2021

Published: 10 June 2021

Citation:

Wenzel M, von Hardenberg J, Welte MN, Doryumu S, Hoeh B,

Wittler C, Höfner T, Kriegmair MC,

Michel MS, Chun FKH, Herrmann J, Mandel $P$ and Westhoff N (2021)

Monoprophylaxis With

Cephalosporins for Transrectal Prostate Biopsy After the

Fluoroquinolone-Era: A MultiInstitutional Comparison of Severe

Infectious Complications.

Front. Oncol. 11:684144.

doi: 10.3389/fonc.2021.684144

\section{Monoprophylaxis With} Cephalosporins for Transrectal Prostate Biopsy After the Fluoroquinolone-Era: A MultiInstitutional Comparison of Severe Infectious Complications

Mike Wenzel ${ }^{1,2,3 *}$, Jost von Hardenberg ${ }^{3,4}$, Maria N. Welte ${ }^{1,3}$, Samuel Doryumu ${ }^{4}$, Benedikt Hoeh ${ }^{1}$, Clarissa Wittler ${ }^{1}$, Thomas Höfner ${ }^{3,5}$, Maximilian C. Kriegmair ${ }^{4}$, Maurice S. Michel ${ }^{4}$, Felix KH. Chun ${ }^{1}$, Jonas Herrmann ${ }^{3,4}$, Philipp Mandel ${ }^{1}$ and Niklas Westhoff ${ }^{3,4}$

1 Department of Urology, University Hospital Frankfurt, Goethe University Frankfurt am Main, Frankfurt, Germany, ${ }^{2}$ Cancer Prognostics and Health Outcomes Unit, Division of Urology, University of Montreal Health Center, Montreal, QC, Canada, ${ }^{3}$ GeSRU Academics Prostate Cancer Working Group, Planegg, Germany, ${ }^{4}$ Department of Urology and Urosurgery, University Medical Center Mannheim, Medical Faculty Mannheim, Heidelberg University, Mannheim, Germany, ${ }^{5}$ Department of Urology, University Hospital Mainz, Mainz, Germany

Background: To compare severe infectious complication rates after transrectal prostate biopsies between cephalosporins and fluoroquinolones for antibiotic monoprophylaxis.

Material and Methods: In the multi-institutional cohort, between November 2014 and July 2020 patients received either cefotaxime (single dose intravenously), cefpodoxime (multiple doses orally) or fluoroquinolones (multiple-doses orally or single dose intravenously) for transrectal prostate biopsy prophylaxis. Data were prospectively acquired and retrospectively analyzed. Severe infectious complications were evaluated within 30 days after biopsy. Logistic regression models predicted biopsy-related infectious complications according to antibiotic prophylaxis, application type and patient- and procedure-related risk factors.

Results: Of 793 patients, 132 (16.6\%) received a single dose of intravenous cefotaxime and were compared to 119 (15\%) who received multiple doses of oral cefpodoxime and 542 (68.3\%) who received fluoroquinolones as monoprophylaxis. The overall incidence of severe infectious complications was $1.0 \%(n=8)$. No significant differences were observed between the three compared groups $(0.8 \%$ vs. $0.8 \%$ vs. $1.1 \%, p=0.9)$. The overall rate of urosepsis was $0.3 \%$ and did not significantly differ between the three compared groups as well.

Conclusion: Monoprophylaxis with third generation cephalosporins was efficient in preventing severe infectious complications after prostate biopsy. Single intravenous dose of cefotaxime and multiday regimen of oral cefpodoxime showed a low incidence of infectious complications $<1 \%$. No differences were observed in comparison to fluoroquinolones.

Keywords: cephalosporins, urosepsis, urinary tract infections, biopsy, prostatic neoplasms, fluoroquinolones 


\section{INTRODUCTION}

Prostate cancer, the most common cancer in men worldwide, is diagnosed with prostate biopsies (1). Currently, two different applicable biopsy approaches are available, a transrectal and transperineal approach (2). Several studies reported that cancer detection rates by systematic biopsies are comparable between both approaches $(3,4)$. However, regarding infectious rates, some studies suggest that the transperineal approach is associated with lower rates of infectious complications, while other report comparable infectious rates (4-6). Nonetheless, sufficient prospective data is lacking. In consequence, the transrectal approach is still used worldwide and guidelines do not recommend one approach over the other, since the advantage of the transrectal approach is the quick and easy performance in an outpatient setting under local anesthesia, while a transperineal biopsy is widely performed under general anesthesia $(2,4)$.

The U.S. Food and Drug Administration in 2018 and the European Medicine Association (EMA) in 2019 suspended the indication for fluoroquinolones as antibiotic prophylaxis due to its toxicity profile (7). In addition, as a consequence of increasing resistance rates, there were more severe infections during the past years after administration of fluoroquinolones (8). Since fluoroquinolones have been the antibiotic prophylaxis of choice for transrectal prostate biopsies for decades, no current guideline recommendation exists for other antimicrobial agents in prophylaxis $(2,9,10)$. Cephalosporins represent an alternative as a monotherapeutic antibiotic prophylaxis. The application type, either as a single intravenous dose or multiple oral doses, has different advantages. However, although several studies investigated appropriate complication rates of other antibiotic regimes after transrectal prostate biopsy, these studies mostly focused on augmented regimes $(11,12)$. In consequence, studies comparing a monotherapeutic prophylaxis with cephalosporins $v s$. fluoroquinolones in a homogenous cohort are still pending (13).

We addressed this void and relied on a multi-institutional prostate biopsy database of two tertiary care university hospitals. We hypothesized that differences according to severe complication rates after transrectal prostate biopsy may not exist in the comparison of cephalosporins vs. fluoroquinolones. Moreover, we hypothesized that application form and duration of the antibiotic prophylaxis does not affect complication rates.

\section{MATERIALS AND METHODS}

\section{Study Population}

The study was conducted in accordance with the Declaration of Helsinki. After ethic committee's approval, patients who underwent a transrectal systematic prostate biopsy or combined magnetic resonance imaging (MRI)-targeted and transrectal systematic biopsy at either the Department of Urology, University Hospital Frankfurt (UKF), Germany or the Department of Urology and Urosurgery, University Medical
Center Mannheim (UMM), Germany, between November 2014 and July 2020 were prospectively acquired and retrospectively analyzed. Exclusion criteria for the subsequent analyses were other antibiotic prophylaxis for transrectal prostate biopsies than cephalosporins or fluoroquinolones or augmented (combination of at least two antibiotic agents) antibiotic regimes $(n=62)$. Indications for prostate biopsies were a primary cancer suspicion or patients under active surveillance in accordance with current guidelines (2).

\section{Transrectal Prostate Biopsy}

According to the former institutional standards, at UMM, a urine culture was taken from every patient prior to prostate biopsies. During the study period, rectal swabs were not obtained by default. At UKF, urine cultures or rectal swabs were not performed on regular basis. Prior to transrectal prostate biopsy, a periprostatic local anesthesia was injected under ultrasoundguidance, as recommended (2). For systematic prostate biopsy, 12 cores (six cores from each prostate lobe) were taken according to current guidelines. For fusion biopsies, at least two cores were taken from each target, with high-end ultrasound machines (University Hospital Frankfurt: HiVison, Hitachi Medical Systems; University Medical Center Mannheim: Artemis ${ }^{\mathrm{TM}}$ ). In addition to targeted biopsy, systematic biopsy was performed in all patients.

\section{Antibiotic Prophylaxis and Follow-Up}

All prostate biopsies were taken under antibiotic prophylaxis with a monotherapy with either cephalosporins or fluoroquinolones. The choice of an antibiotic regimen was based on the institutional standard at the time of biopsy. Since bioavailability may differ between the application types of both third generation cephalosporins, tabulation was made for cefotaxime (single dose intravenous application of $2 \mathrm{~g} \mathrm{20-60}$ minutes prior to biopsy) vs. cefpodoxime (multiple doses of $200 \mathrm{mg}$ oral application twice daily, beginning at least 24 hours prior to biopsy for five days according to current recommendations (14)). Due to the comparable bioavailability between oral and intravenous application, this stratification was not made for fluoroquinolones (intravenous or oral five-day application according to the historical fluoroquinolone standard). Patients at risk for an infectious endocarditis received an agent active against enterococci and were not included in this analysis. Patient and tumor characteristics, as well as severe infectious complication rates, defined as an emergency hospital consultation due to an UTI (according to current guidelines (14)) with or without fever, were collected from the patients' hospital files within 30 days after prostate biopsy. Urosepsis was defined as previously described (15). During the pre-interventional briefing, patients were routinely instructed to inform a urologist in case of relevant complications.

\section{Statistical Analysis}

Descriptive statistics included frequencies and proportions for categorical variables. Medians and interquartile ranges (IQR) were reported for continuously coded variables. The Chi-square test was used for statistical significance in proportions 
differences. The t-test and Kruskal-Wallis test examined the statistical significance of means' and distributions' differences.

All tests were two sided with a level of significance set at $\mathrm{p}<0.05$ and $\mathrm{R}$ software environment for statistical computing and graphics (version 3.4.3) was used for all analyses.

\section{RESULTS}

\section{Patient and Procedure Characteristics}

Overall, 793 patients were eligible for analyses. The median age was 66 years (IQR 61-72 years). Among all patients, 66.7\% were biopsy naïve. A median number of 14 cores (IQR 13-15) per biopsy were obtained. In total, 36 patients $(4.5 \%)$ had diabetes mellitus, 33 patients $(4.2 \%)$ had at least three chronical diseases (defined as multimorbidity), 16 patients (2\%) were immunosuppressed, nine patients (1.1\%) had an indwelling catheter and six patients $(0.8 \%)$ reported recurrent UTIs. In the group of patients who received multiple oral doses of cefpodoxime, significantly more patients had comorbidities $(\mathrm{p}<0.001)$. An UTI during the past twelve months was reported by nine patients $(1.1 \%)$ and 50 patients received an antibiotic treatment for any cause within the last six months prior to biopsy (6.3\%).

Significant differences between the subgroups of antibiotic regimens existed in the median number of cores per biopsy, a history of UTIs within the last 12 months and application of antibiotics within the last six months, as well as cancer detection rates (all $\mathrm{p}<0.05)$.

All patient characteristics and biopsy results are displayed in Table 1.

TABLE 1 | Patient characteristics stratified by antibiotic prophylaxis for transrectal prostate biopsy.

\begin{tabular}{|c|c|c|c|c|c|c|}
\hline Variable & & Overall $\mathrm{N}=793$ & $\begin{array}{c}\text { Cefotaxime single dose } \\
\text { (intravenous) } \mathrm{N}=132(16.6 \%)\end{array}$ & $\begin{array}{l}\text { Cefpodoxime multiple } \\
\text { doses(oral) } \mathrm{N}=119 \\
(15.0 \%)\end{array}$ & $\begin{array}{c}\text { Fluoroquinolones } \\
\mathrm{N}=542(68.3 \%)\end{array}$ & $\begin{array}{c}P \\
\text { value }\end{array}$ \\
\hline Age, years & Median (IQR) & $66(61-72)$ & $66(60-73)$ & 66 (60-72) & 67 (61-72) & 0.7 \\
\hline PSA, ng/ml & Median (IQR) & $7.3(5.3-11.9)$ & $7.0(5.2-9.6)$ & $7.4(5.0-12.2)$ & $7.4(5.3-12.2)$ & 0.1 \\
\hline $\begin{array}{l}\text { Prostate volume, } \\
\mathrm{ml}\end{array}$ & Median (IQR) & $50(38-70)$ & 58 (43-73) & 50 (36-72) & 50 (38-70) & 0.8 \\
\hline $\begin{array}{l}\text { Biopsy positive, } \\
\text { n (\%) }\end{array}$ & Yes & $516(65.1)$ & $95(72.0)$ & $84(70.6)$ & 337 (62.2) & 0.04 \\
\hline Cores per biopsy & Median (IQR) & $14(13-15)$ & $16(14-16)$ & $13(12-14)$ & $14(12-15)$ & $<0.001$ \\
\hline $\begin{array}{l}\text { Positive cores per } \\
\text { biopsy }\end{array}$ & Median (IQR) & $2(0-6)$ & $3(0-6)$ & $3(0-5)$ & $2(0-6)$ & 0.2 \\
\hline Core ratio in \% & Median (IQR) & $40(20-50)$ & $30(20-40)$ & $40(20-50)$ & $30(20-60)$ & 0.05 \\
\hline \multirow[t]{2}{*}{ Hospital, n (\%) } & UKF & 441 (55.6) & $0(0)$ & 99 (83.2) & $342(63.1)$ & \\
\hline & UMM & $352(44.4)$ & $132(100)$ & $20(16.8)$ & 200 (36.9) & \\
\hline DRE, n (\%) & suspicious & $214(27)$ & $33(25)$ & $32(26.9)$ & $149(27.5)$ & 0.9 \\
\hline \multirow{4}{*}{$\begin{array}{l}\text { Previous biopsies, } \\
\text { n (\%) }\end{array}$} & 0 & $529(66.7)$ & $83(62.9)$ & 84 (70.6) & $362(66.8)$ & 0.6 \\
\hline & 1 & $182(23)$ & $30(22.7)$ & $27(22.7)$ & $125(23.1)$ & \\
\hline & 2 & $56(7.1)$ & $13(9.8)$ & $5(4.2)$ & $38(7.0)$ & \\
\hline & $\geq 3$ & $26(3.3)$ & $6(4.5)$ & $3(2.5)$ & $17(3.1)$ & \\
\hline \multirow{5}{*}{$\begin{array}{l}\text { Comorbidities, } \\
\text { n (\%) }\end{array}$} & Diabetes & $36(4.5)$ & $6(4.5)$ & $14(11.8)$ & $16(3.0)$ & $<0.001$ \\
\hline & Immunosuppression & $16(2.0)$ & $0(0)$ & $10(8.4)$ & $6(1.1)$ & \\
\hline & Catheter & $9(1.1)$ & $0(0)$ & $2(1.7)$ & 7 (1.3) & \\
\hline & Multimorbidity & $33(4.2)$ & $11(8.3)$ & $2(1.7)$ & $20(3.7)$ & \\
\hline & Recurrent UTI & $6(0.8)$ & $0(0)$ & $3(2.5)$ & $3(0.6)$ & \\
\hline $\begin{array}{l}\text { Rectal swab prior } \\
\text { to biopsy, n (\%) }\end{array}$ & Yes & $32(4.0)$ & $10(7.6)$ & $8(6.7)$ & $14(2.6)$ & $<0.001$ \\
\hline $\begin{array}{l}\text { Urine culture prior } \\
\text { to biopsy, } \mathrm{n}(\%)\end{array}$ & Yes & $368(46.4)$ & $132(100)$ & $31(26.1)$ & $205(37.8)$ & $<0.001$ \\
\hline $\begin{array}{l}\text { Urine culture } \\
\text { positive prior to } \\
\text { biopsy, } n(\%)\end{array}$ & Yes & $45(5.7)$ & $15(11.4)$ & $3(2.5)$ & $27(5.0)$ & 0.9 \\
\hline $\begin{array}{l}\text { Histologically } \\
\text { confirmed } \\
\text { prostatitis, n (\%) }\end{array}$ & Yes & 266 (33.5) & $74(56.1)$ & 42 (35.3) & $150(27.7)$ & 0.6 \\
\hline $\begin{array}{l}\text { UTI within last } 12 \\
\text { months, n (\%) }\end{array}$ & Yes & $9(1.1)$ & $1(0.8)$ & $5(4.2)$ & $3(0.6)$ & $<0.001$ \\
\hline $\begin{array}{l}\text { Antibiotics within } \\
\text { last } 6 \text { months, } \\
\text { n (\%) }\end{array}$ & Yes & $50(6.3)$ & $16(12.1)$ & $10(8.4)$ & $24(4.4)$ & $<0.001$ \\
\hline
\end{tabular}

Descriptive characteristics of 793 patients who underwent transrectal prostate biopsy stratified according to prescribed antibiotic prophylaxis and single dose (intravenous) or multiple doses (oral) application. PSA, initial Prostate Specific Antigen; DRE, Digital rectal examination; UTI, Urinary tract infection; UKF, University Hospital Frankfurt; UMM, University Hospital Mannheim. 


\section{Incidence of Infectious Complications}

A single dose of cefotaxime was administered intravenously to 132 patients (16.6\%), whereas 119 patients (15\%) received multiple oral doses of cefpodoxime. Both groups were compared to 542 patients (68.3\%) who received fluoroquinolones either as an intravenous single dose $(28.4 \%)$ or multiple oral doses $(71.6 \%)$ prophylaxis. A multiple dose approach was applied for a median of five days (IQR 5-5) for oral cephalosporins and fluoroquinolones.

The total number of patients with severe infectious complications after biopsy was eight (1.0\%). One patient per cephalosporine group $(0.8 \%$ each) and six patients $(1.1 \%)$ in the fluoroquinolone group reported a complication $(\mathrm{p}=0.9)$. The rate of urosepsis was $0.3 \%(n=2)$ including one patient in the cefotaxime group and one patient in the fluoroquinolone group. Two patients with an UTI $(0.4 \%)$ and three patients with a prostatitis $(0.6 \%)$ received a prophylaxis with fluoroquinolones, one patient with an epididymitis $(0.8 \%)$ received cefpodoxime. However, there were no significant differences regarding the single infectious complications $(\mathrm{p}=0.3)$.

Moreover, according to fever after biopsy, no significant differences were detected between all groups $(\mathrm{p}=0.6)$. Table 2 summarizes infectious complications and treatments.

\section{DISCUSSION}

Due to the increasing bacterial resistance rates of fluoroquinolones, reported to be up to $50 \%$ in Escherichia coli, and the suspended indication for prophylaxis due to rare, but potentially severe side effects (e.g., confusion, arterial aneurysms, tendinopathy), a paradigm shift in antibiotic prophylaxis for prostate biopsies is required $(9,16)$. We aimed to address this void and revealed several important observations:

First, the results of this large multicenter retrospective analysis of patients undergoing a transrectal prostate biopsy demonstrated a low rate of clinically relevant overall infectious complications (1.0\%). Moreover, no significant differences between the usage of cephalosporins vs. fluoroquinolones as a monotherapeutic antibiotic prophylaxis were observed $(0.8 \% v s$. $1.1 \%, \mathrm{p}=0.9)$. These observations are noteworthy, since in a recent meta-analysis among 1141 evaluating antibiotic prophylaxis vs. placebo, the rate of infectious complications was $5.6 \%$ (10). The lower rate in our cohort might be explained by the definition of infectious complications. We focused exclusively on complications leading to an emergency department visit. Since most studies did not distinguish between the severity of infectious complications, inclusion of e.g., a mild cystitis led to higher rates of overall complications. Importantly, the incidence of complications also depends on geographic regions which results in variation of complication rates from $0-6 \%$, as reported in the systematic review of Roberts et al. (17).

Second, the pathophysiology of post-biopsy infectious complications is explained by two mechanisms: Firstly, flora of the large bowel is directly translocated into the prostate including Escherichia coli as the most frequent causative microorganism (70-90\%) and secondly, a bacterial colonization of the prostate or urogenital mucosa before the procedure is considered to cause an UTI afterwards $(18,19)$. By now, there is an ongoing debate on the optimal alternative non-fluoroquinolone antibiotic regimens to avoid post-biopsy complications. The current European Urology Position Paper on the Prevention of Infectious Complications recommends performing a transperineal biopsy

TABLE 2 | Infectious complication related to the antibiotic prophylaxis regimen.

\begin{tabular}{|c|c|c|c|c|c|c|}
\hline Variable & & $\begin{array}{l}\text { Overall } \\
\mathrm{N}=793\end{array}$ & $\begin{array}{c}\text { Cefotaxime single dose } \\
\text { (intravenous) } \mathrm{N}=132(16.6 \%)\end{array}$ & $\begin{array}{l}\text { Cefpodoxime multiple doses } \\
\text { (oral) } \mathrm{N}=119(15 \%)\end{array}$ & $\begin{array}{l}\text { Fluoroquinolones } \\
\mathrm{N}=542(68.3 \%)\end{array}$ & $\begin{array}{c}P \\
\text { value }\end{array}$ \\
\hline $\begin{array}{l}\text { Duration of antibiotic } \\
\text { prophylaxis, days }\end{array}$ & $\begin{array}{l}\text { Median } \\
(\mathrm{IQR})\end{array}$ & $5(1-5)$ & $1(1-1)$ & $5(5-5)$ & $5(1-5)$ & \\
\hline \multirow[t]{2}{*}{ Application of prophylaxis, $n(\%)$} & intravenous & $\begin{array}{c}176 \\
(22.2)\end{array}$ & $132(100)$ & $0(0)$ & $44(8.1)$ & \\
\hline & oral & $\begin{array}{c}577 \\
(72.8)\end{array}$ & $0(0)$ & $119(100)$ & $458(84.5)$ & \\
\hline \multirow[t]{4}{*}{ Infectious complication, $\mathrm{n}(\%)$} & Epididymitis & $1(0.1)$ & $0(0)$ & $1(0.8)$ & $0(0)$ & 0.3 \\
\hline & UTI & $2(0.3)$ & $0(0)$ & $0(0)$ & $2(0.4)$ & \\
\hline & Prostatitis & $3(0.4)$ & $0(0)$ & $0(0)$ & $3(0.6)$ & \\
\hline & Urosepsis & $2(0.3)$ & $1(0.8)$ & $0(0)$ & $1(0.2)$ & \\
\hline Fever after biopsy, n (\%) & Yes & $5(0.6)$ & $1(0.8)$ & $0(0)$ & $4(0.7)$ & 0.6 \\
\hline $\begin{array}{l}\text { Antibiotic treatment of infectious } \\
\text { complication, } n(\%)\end{array}$ & oral & $4(0.5)$ & $0(0)$ & $1(0.8)$ & $3(0.6)$ & \\
\hline $\begin{array}{l}\text { Duration of infect treatment, } \\
\text { days }\end{array}$ & $\begin{array}{l}\text { Median } \\
\text { (IQR) }\end{array}$ & $10(6-13)$ & $11(11-11)$ & $5(5-5)$ & $11(7-16)$ & 0.7 \\
\hline
\end{tabular}

Antibiotic prophylaxis, infectious complication rates and infect treatment of 793 patients who underwent transrectal prostate biopsy stratified according to the prescribed antibiotic prophylaxis application form. UTI: Urinary tract infection. 
whenever possible (20). If not feasible, three ways of antibiotic prophylaxis for transrectal biopsy are available: i) targeted prophylaxis based on a rectal swab or stool culture, ii) augmented prophylaxis with a combination of at least two different classes of antibiotics or iii) empirical monotherapeutic alternatives to fluoroquinolones (20). Since no superiority of an augmented prophylaxis has yet been demonstrated by ten previous published randomized controlled trials, a monoprophylaxis presents a safe strategy at present. Moreover, since rectal swabs are not available everywhere, an optimal empirical treatment has to be defined. In consequence, our data suggest that third generation cephalosporins, intravenously or orally administered, represent a safe empirical treatment strategy in accordance with the current European Urology Position Paper.

Third, cephalosporins of the third generation, a class of $B$ lactam antibiotics, have a broad-spectrum antimicrobial activity against gram-positive, but more relevant gram-negative organisms and are therefore suitable candidates for prostate biopsy prophylaxis. Concerns against their usage have been made since resistancies against $ß$-lactams in gram-negative pathogens may lead to failure of prophylaxis (21). Nonetheless, the low incidence of severe infectious complications in our cohort strengthens the evidence for the appropriate use of cephalosporins, either orally or intravenously administered, in transrectal prostate biopsy. This observation is in an agreement with four RCTs, which investigated complication rates of cephalosporins (cefuroxime, cefixime or ceftriaxone) vs. fluoroquinolones or piperacillin/tazobactam (2225). Moreover, in a pooled analysis of three of these studies, including 244 men receiving non-cephalosporins vs. 254 men receiving cephalosporins, no statistically significant differences were detected regarding infectious complication and hospitalization rates (RR 0.57, 95\% CI 0.12-2.63). Additionally, the same meta-analysis compared 14 studies of nonfluoroquinolones vs fluoroquinolones, where significantly less infectious events were observed with non-fluoroquinolones prophylaxis (8). However, it is of note that consideration of the local resistance patterns increases the safety of cephalosporins since resistance varies widely depending on the geographical region. A rectal swab or stool culture prior to biopsy to detect resistances beforehand and perform a targeted therapy showed significantly lower infection rates compared to an empirical fluoroquinolone prophylaxis (RR 2.1, 95\% CI 1.53-2.88) although data on non-fluoroquinolones-targeted prophylaxes is lacking (10). Due to the former institutional standards, only 32 patients had received a rectal swab. None of these patients had an infectious complication, independent of the antibiotic prophylaxis. Those study results and our observations may be indicative for a general performance of rectal swabs.

With respect to other monotherapeutic antibiotic prophylaxis options, the European Commission recommended 2020 a fosfomycin trometamol usage for prophylaxis in men undergoing prostate biopsy (26). Promising results were demonstrated in different meta-analyses, although one large case-control study revealed inferiority compared to ciprofloxacin $(27,28)$. Consequently, the definite effect of fosfomycin trometamol remains under debate and no recent trial compared cephalosporins vs. fosfomycin trometamol yet.
Further trials or network meta-analyses are needed to directly compare the promising results of cephalosporins vs. fosfomycin for transrectal prostate biopsy.

Fourth, we also assessed whether the application type and duration of the cephalosporins affected the occurrence of severe infectious complications. Although urosepsis occurred in one patient in the single dose group and epididymitis in one patient in the multiple doses group, we observed no significant differences between the application types according to overall infections. This result is contradictory to the recent meta-analysis by Pilatz et al. The available studies on fluoroquinolones indicate that a 1-day prophylaxis beginning at least 24 hours prior to biopsy is comparable to a 3-day course, whereas a single-shot prophylaxis less than 24 hours prior to biopsy is inferior compared to a longer course (10). However, this recommendation was not corroborated in a Cochrane review and data mainly relied on fluoroquinolones (29). In consequence, with regard to duration and application type, a comparison to our results cannot be made.

The different antibiotic strategies of both tertiary care hospitals demonstrated comparable efficacy in prevention of severe infectious complications in this study. Whereas from an antibiotic stewardship point of view a single dose prophylaxis is especially beneficial to avoid antibiotic resistances, the advantage of the multiple oral doses' application might be extended drug levels. Despite pharmacokinetic differences, patients might prefer the oral application over an intravenous access or vice versa.

Limitations of this work are firstly the non-randomized retrospective cohort design, which nevertheless increases the knowledge on usage of cephalosporins as a monoprophylaxis due to its large multicenter population size. Second, infectious complications were not assessable from outpatient visits, meaning that mild complications might be underestimated. Moreover, small numbers of infectious complications precluded more complex analyses, such as logistic regression models. Third, definitions of UTI complications were not based on urine cultures but on self-reported symptoms and in-hospital examinations and reports. Finally, despite the large cohort size, it is likely that the low incidence of complication events limited the statistical power of some variables of interest and the majority of patients received fluoroquinolones. Thus, especially the evaluation of other risk factors or comorbidities associated with infectious complications was unfortunately not possible.

\section{DATA AVAILABILITY STATEMENT}

The raw data supporting the conclusions of this article will be made available by the authors, without undue reservation.

\section{ETHICS STATEMENT}

The studies involving human participants were reviewed and approved by the Department of Urology, University Hospital Frankfurt (UKF), Germany and the Department of Urology and Urosurgery, University Medical Center Mannheim (UMM). 


\section{AUTHOR CONTRIBUTIONS}

Conceptualization: MW, JH, PM, and NW. Data curation: MW, JH, MNW, SD, BH, CW, PM, and NW. Formal analysis: MW, JH, PM,

\section{REFERENCES}

1. Siegel RL, Miller KD, Jemal A. Cancer Statistics, 2019. CA Cancer J Clin (2019) 69(1):7-34. doi: 10.3322/caac.21551

2. Mottet N, Cornford P, Van den Bergh RCN, Briers E, De Santis M, Fanti S, et al. EAU Guidelines. Edn. Presented at the EAU Annual Congress Amsterdam 2020 (2020).

3. Xue J, Qin Z, Cai H, Zhang C, Li X, Xu W, et al. Comparison Between Transrectal and Transperineal Prostate Biopsy for Detection of Prostate Cancer: A Meta-Analysis and Trial Sequential Analysis. Oncotarget (2017) 8(14):23322-36. doi: 10.18632/oncotarget.15056

4. Xiang J, Yan H, Li J, Wang X, Chen H, Zheng X. Transperineal Versus Transrectal Prostate Biopsy in the Diagnosis of Prostate Cancer: A Systematic Review and Meta-Analysis. World J Surg Oncol (2019) 17(1):31. doi: 10.1186/ s12957-019-1573-0

5. Wajswol E, Winoker JS, Anastos H, Falagario U, Okhawere K, Martini A, et al. A Cohort of Transperineal Electromagnetically Tracked Magnetic Resonance Imaging/Ultrasonography Fusion-Guided Biopsy: Assessing the Impact of Inter-Reader Variability on Cancer Detection. BJU Int (2020) 125 (4):531-40. doi: 10.1111/bju.14957

6. Winoker JS, Wajswol E, Falagario U, Maritini A, Moshier E, Voutsinas N, et al. Transperineal Versus Transrectal Targeted Biopsy With Use of Electromagnetically-Tracked Mr/Us Fusion Guidance Platform for the Detection of Clinically Significant Prostate Cancer. Urology (2020) 146:27886. doi: 10.1016/j.urology.2020.07.072

7. Bonkat G, Pilatz A, Wagenlehner F. Time to Adapt Our Practice? The European Commission Has Restricted the Use of Fluoroquinolones Since March 2019. Eur Urol (2019) 76(3):273-5. doi: 10.1016/j.eururo.2019. 06.011

8. Liss MA, Ehdaie B, Loeb S, Meng MV, Raman JD, Spears V, et al. An Update of the American Urological Association White Paper on the Prevention and Treatment of the More Common Complications Related to Prostate Biopsy. J Urol (2017) 198(2):329-34. doi: 10.1016/j.juro.2017.01.103

9. European Medical Association. Disabling and Potentially Permanentside Effects Lead to Suspension or Restrictionsof Quinolone and Fluoroquinolone Antibiotics (2019). Available at: https://www.ema.europa.eu/en/documents/ referral/quinolone-fluoroquinolone-article-31-referral-disabling-potentiallypermanent-side-effects-lead_en.pdf.

10. Pilatz A, Dimitropoulos K, Veeratterapillay R, Yuan Y, Omar MI, MacLennan S, et al. Antibiotic Prophylaxis for the Prevention of Infectious Complications Following Prostate Biopsy: A Systematic Review and Meta-Analysis. J Urol (2020) 204(2):224-30. doi: 10.1097/JU. 0000000000000814

11. Luong B, Danforth T, Visnjevac O, Suraf M, Duff M, Chevli KK. Reduction in Hospital Admissions With the Addition of Prophylactic Intramuscular Ceftriaxone Before Transrectal Ultrasonography-Guided Prostate Biopsies. Urology (2015) 85(3):511-6. doi: 10.1016/j.urology.2014.10.047

12. Adamczyk P, Juszczak K, Prondzinska M, Kędzierska A, Szwajkert-Sobiecka $\mathrm{H}$, Drewa T. Fluoroquinolone-Resistant Escherichia Coli in Intestinal Flora of Patients Undergoing Transrectal Ultrasound-Guided Prostate Biopsy Possible Shift in Biopsy Prophylaxis. Cent Eur J Urol (2017) 70(2):192-6. doi: $10.5173 /$ ceju.2017.739

13. Wenzel M, Welte MN, Theissen LH, Wittler C, Hoeh B, Humke C, et al. Comparison of Complication Rates With Antibiotic Prophylaxis With Cefpodoxime Versus Fluoroquinolones After Transrectal Prostate Biopsy. Eur Urol Focus (2020). doi: 10.1016/j.euf.2020.11.006

14. Interdisziplinä Epidemiologie, Diagnostik, Therapie Prävention Unkompliziert Ererworbener Harnwegsinfektionen Bei Erwachsenen (2017). Available at: https://www.awmf.org/uploads/tx_szleitlinien/0430441_S3_ Harnwegsinfektionen_2017-05.pdf. and NW. Funding acquisition: - Investigation: MW, JH, PM, and NW. Methodology: MW, PM, and NW. Supervision: TH, MK, MM, and FC. Validation: TH, MK, MM, FC, JH, PM, and NW. All authors contributed to the article and approved the submitted version.

15. Wagenlehner FME, Pilatz A, Weidner W. Urosepsis-From the View of the Urologist. Int J Antimicrob Agents (2011) 38(Suppl):51-7. doi: 10.1016/ j.ijantimicag.2011.09.007

16. European Centre for Disease Prevention and Control. Surveillance of Antimicrobial Resistance in Europe - Annual Report of the European Antimicrobial Resistance Surveillance Network (Eras-Net). Stockholm: ECD (2017). Available at: https://ecdc.europa.eu/sites/portal/files/documents/ AMR-surveillance-EARS-Net-2017.pdf.

17. Roberts MJ, Bennett HY, Harris PN, Holmes M, Grummet J, Naber K, et al. Prostate Biopsy-related Infection: A Systematic Review of Risk Factors, Prevention Strategies, and Management Approaches. Urology (2017) 104:11-21. doi: 10.1016/j.urology.2016.12.011

18. Williamson DA, Barrett LK, Rogers BA, Freeman JT, Hadway P, Paterson DL Infectious Complications Following Transrectal Ultrasound-Guided Prostate Biopsy: New Challenges in the Era of Multidrug-Resistant Escherichia Coli. Clin Infect Dis Off Publ Infect Dis Soc Am (2013) 57(2):267-74. doi: 10.1093/ $\mathrm{cid} / \mathrm{cit} 193$

19. Zowawi HM, Harris PNA, Roberts MJ, Tambyah PA, Schembri MA Pezzani MD, et al. The Emerging Threat of Multidrug-Resistant Gramnegative Bacteria in Urology. Nat Rev Urol (2015) 12(10):570-84. doi: 10.1038/nrurol.2015.199

20. Pilatz A, Veeratterapillay R, Dimitropoulos K, Omar MI, Pradere B, Yuan Y, et al. European Association of Urology Position Paper on the Prevention of Infectious Complications Following Prostate Biopsy. Eur Urol (2021) 79 (1):11-5. doi: 10.1016/j.eururo.2020.10.019

21. Azap OK, Arslan H, Serefhanoğlu K, Colakoğlu S, Erdoğan H, Timurkaynak F, et al. Risk Factors for Extended-Spectrum Beta-Lactamase Positivity in Uropathogenic Escherichia Coli Isolated From CommunityAcquired Urinary Tract Infections. Clin Microbiol Infect Off Publ Eur Soc Clin Microbiol Infect Dis (2010) 16(2):147-51. doi: 10.1111/j.1469-0691.2009. 02941.x

22. Cam K, Kayikci A, Akman Y, Erol A. Prospective Assessment of the Efficacy of Single Dose Versus Traditional 3-Day Antimicrobial Prophylaxis in 12-Core Transrectal Prostate Biopsy. Int J Urol Off J Jpn Urol Assoc (2008) 15(11):9971001. doi: 10.1111/j.1442-2042.2008.02147.x

23. Brewster SF, MacGowan AP, Gingell JC. Antimicrobial Prophylaxis for Transrectal Prostatic Biopsy: A Prospective Randomized Trial of Cefuroxime Versus Piperacillin/Tazobactam. Br J Urol (1995) 76(3):351-4. doi: 10.1111/ j.1464-410x.1995.tb07713.x

24. Samarinas M, Skriapas K AK. Efficacy of Prophylactic Administration of Prurifloxacin vs. Cefixime in Patients Undergoing Ultrasound Guided Prostate Biopsy: A Prospective Randomized Study. Eur Urol Suppl (2019) 18:e118. doi: 10.1016/S1569-9056(19)30088-0

25. Pipitpanpipit T, Sntanirand P, Kongchareonsombat K. A Comparative Study of Oral Medication to Prevent Transient Bacteriemia and Adverse Events From Transrectal Prostatic Biopsy: Ciprofloxacin Versus Cefixime. J Med Assoc Thailand (2017) 100:528.

26. Recommendations to Restrict Use of Fosfomycin Antibiotics. Available at: https://www.ema.europa.eu/en/documents/referral/fosfomycin-article-31referral-recommendations-restrict-use-fosfomycin-antibiotics_en.pdf.

27. Carignan A, Sabbagh R, Masse V, Gagnon N, Montpetit LP, Smith MA, et al Effectiveness of Fosfomycin Tromethamine Prophylaxis in Preventing Infection Following Transrectal Ultrasound-Guided Prostate Needle Biopsy: Results From a Large Canadian Cohort. J Glob Antimicrob Resist (2019) 17:112-6. doi: 10.1016/j.jgar.2018.11.020

28. Noreikaite J, Jones P, Fitzpatrick J, Amitharaj R, Pietropaolo A, Vasdev N, et al. Fosfomycin vs. Quinolone-Based Antibiotic Prophylaxis for Transrectal Ultrasound-Guided Biopsy of the Prostate: A Systematic Review and MetaAnalysis. Prostate Cancer Prostatic Dis (2018) 21(2):153-60. doi: 10.1038/ s41391-018-0032-2 
29. Zani EL, Clark OAC, Rodrigues Netto N. Antibiotic Prophylaxis for Transrectal Prostate Biopsy. Cochrane Database Syst Rev (2011) (5): CD006576. doi: 10.1002/14651858.CD006576.pub2

Conflict of Interest: The authors declare that the research was conducted in the absence of any commercial or financial relationships that could be construed as a potential conflict of interest.
Copyright (ङ 2021 Wenzel, von Hardenberg, Welte, Doryumu, Hoeh, Wittler, Höfner, Kriegmair, Michel, Chun, Herrmann, Mandel and Westhoff. This is an open-access article distributed under the terms of the Creative Commons Attribution License (CC BY). The use, distribution or reproduction in other forums is permitted, provided the original author(s) and the copyright owner(s) are credited and that the original publication in this journal is cited, in accordance with accepted academic practice. No use, distribution or reproduction is permitted which does not comply with these terms. 